\title{
Multifocal electroretinographical changes in monkeys with experimental ocular hypertension: a longitudinal study
}

\author{
Jan Kremers · Arno Doelemeyer • \\ Elzbieta A. Polska · Fabrice Moret • \\ Christian Lambert · George N. Lambrou
}

Received: 10 April 2007/Accepted: 21 November 2007/Published online: 1 January 2008

(C) Springer-Verlag 2007

\begin{abstract}
Purpose To study the time course of changes in the multifocal electroretinograms (mfERG) in monkeys with experimental ocular hypertension (OHT). Methods The mfERGs were recorded in 12 eyes out of 6 monkeys. Two baseline measurements were used to quantify the reproducibility, the inter-ocular and the inter-individual variability of the ERG signals. Thereafter, the trabeculum of one eye of each animal was lasercoagulated in one to three sessions to induce OHT.
\end{abstract}

J. Kremers - A. Doelemeyer · F. Moret .

C. Lambert · G. N. Lambrou

Novartis Institutes for Biomedical Research, 4002 Basel,

Switzerland

Present Address:

J. Kremers $(\square)$

Department of Ophthalmology, University of

Erlangen-Nuremberg, Schwabachanlage 6,

91054 Erlangen, Germany

e-mail: jan.kremers@uk-erlangen.de

J. Kremers

Department of Optometry, School of Life Sciences,

University of Bradford, Bradford, UK

E. A. Polska

Department of Clinical Pharmacology, Medical

University of Vienna, Vienna, Austria

Present Address:

F. Moret

University of Freiburg Eye Hospital, 79106 Freiburg,

Germany
ERG measurements were repeated regularly in a period of 18 months and the changes in ERG waveforms were quantified. Results All animals displayed OHT (between 20 and $50 \mathrm{mmHg}$ ) in the laser-coagulated eyes. An ERG change was defined as the sum of differences during the first $90 \mathrm{~ms}$ between the laser-coagulated eye and the same eye before laser coagulation and between the lasercoagulated eye and the non-treated fellow eye. Three animals displayed significant changes for nearly all retinal areas and all stimulus conditions. The three remaining animals displayed significant changes only in one comparison, indicating very mild changes. The data indicate that a high stimulus contrast is more sensitive to detect changes, probably because of a better signal-to-noise ratio. Moreover, the comparisons with the fellow eye are more sensitive to detect changes than comparisons with the measurements before laser-coagulation. Conclusions OHT does not always lead to ERG changes. Comparisons with fellow eyes using high contrast stimuli are more sensitive to detect changes related to OHT.

Keywords Glaucoma .

Multifocal electroretinograms .

Non-human primates - Ocular hypertension
Abbreviations
IOP Intraocular pressure
OHT Ocular hypertension 


\section{Introduction}

Many attempts have been made to detect early glaucomatous retinal changes with electroretinography (ERG; [1-3]), because the ERG is one of the few objective non-invasive methods that can be used in a clinical environment to test retinal function. Furthermore, the ERG can be used in studies on animal models of glaucoma, so that the mechanisms of action of the disease and possible methods of intervention can be studied.

Several stimulus techniques have been employed to describe alterations in the retinal functional organization. Ganzfeld flash ERGs have been used [4] to describe glaucoma-induced changes in different ERG components. Pattern ERGs are thought to mainly reflect activity in the inner retina [5]. The pattern ERG has been used extensively to detect glaucoma related functional changes $[6,7]$. With the introduction of the multifocal ERG [8] (mfERG) it became possible to obtain a spatial distribution of the retinal responses. Since then, the mfERG was used to detect possible glaucoma-related retinal damage that were spatially localized [2, 9-15].

Till date only a few detailed longitudinal studies of the alterations in ERG responses in primates after the introduction of ocular hypertension, followed over an extended time, are available $[10,16]$. There are several studies from Harwerth, Frishman and colleagues in which the long-lasting effect of ocular hypertension on visual fields, the normal and slow sequence mfERG and the photopic negative response in the ERG and their correlations were studied [17-19]. It is the purpose of the present study to provide a description of the changes in the mfERG after experimentally induced ocular hypertension (OHT) and to correlate them with the intraocular pressure (IOP). In addition, the present study gives an overview of the reproducibility, the inter-individual variability and the inter-ocular variability of the mfERG signals similar to those presented previously for the Ganzfeld ERG and the pattern ERG in non-human primates [20, 21]. Furthermore, the influence of stimulus contrasts on the sensitivity to detect electrophysiological changes is studied, because there are indications that distinct ERG components may be more pronounced under certain contrast conditions. OHT induced changes may therefore be more visible when contrasts are used that enhance those components that are specially vulnerable to glaucomatous changes $[11,14$, $15,22]$. Detection of electrophysiological changes can be based upon differences with the responses in the same eye before OHT induction or with the responses in the fellow eye. The sensitivities of these analysis methods are compared. Finally, it was studied whether different retinal areas have distinct susceptibilities to OHT.

\section{Methods}

Animals

The experiments were performed on six adult male macaques (Macaca fascicularis). All experimental and animal care procedures adhered to the ARVO Statement for the Use of Animals in Ophthalmic and Vision Research and were reviewed by the local veterinary authorities. Ocular hypertension was induced by photocoagulation of the trabecular meshwork (similar to the method described by Raz et al. [14]). Briefly, before laser-coagulation, the animals were sedated by intramuscular injections of Ketamin $(10 \mathrm{mg} / \mathrm{kg})$. The entire trabecular meshwork of one eye was coagulated using an argon laser by approximately 50-65 evenly distributed laser spots of $75-100 \mu \mathrm{m}$ size $(1.1 \mathrm{~W} ; 0.5 \mathrm{~s})$. In all animals coagulations were performed at day 0 . In one animal (G7) laser-coagulation was repeated 136 days after the first session. Two animals received two additional laser-coagulations (G8: 136 and 193 days after the first session; M7: 73 and 136 days after the first session).

\section{Protocol}

The mfERGs were measured from both eyes of each animal in several sessions over a period of about 18 months. Before the induction of ocular hypertension by laser-coagulation of the trabecular meshwork, the mfERGs were recorded in two baseline measurements. In the first 4 months after the first laser-coagulation, mfERGs were measured every second week. Thereafter, the intervals were increased for those animals for which a steady state was assumed to be reached (when the IOPs and the 
ERGs did not change in four consecutive measurements). The ERG recordings described in the present article were part of a more extended study in which morphological measurements (including Optical Coherence Tomography (OCT), scanning laser polarimetry (GDx), and confocal scanning laser tomography) were also performed. Preliminary results of these studies are presented elsewhere [23-25]. The present article is only concerned with the results of the electrophysiological measurements. An experimental session, in which all ERG recordings and morphological measurements were performed, lasted about $6 \mathrm{~h}$. The intraocular pressures (IOPs) of the two eyes were measured at the beginning, the end and at two different times during these sessions using a calibrated tonometer (TonoPen XL; Mentor Ophthalmics Inc., Norwell, MA, USA).

Before mfERG measurements were performed, the animals were sedated by intramuscular injections of ketamin $(15 \mathrm{mg} / \mathrm{kg})$ after which the first IOP measurements were performed. Anesthesia was obtained with a continuous intravenous infusion of propofol $(5 \mathrm{mg} /(\mathrm{kg} \mathrm{h}))$. The infusion rate of propofol has a significant effect on the amplitude of the b-wave in dogs [26], where, however, much higher infusion rates were used. In pigs, propofol anesthesia has less influence on the ERG when compared with the barbiturate thiopentone [27]. We have no observations on changes in the measured waveforms in the monkey experiments. However, we used a fixed infusion rate in the experiments. Therefore, any changes in waveform are likely to be induced by other factors. Muscle relaxation was obtained through the intravenous administration of an initial dose of $0.1 \mathrm{mg} / \mathrm{kg}$ vecuronium and additional doses of $0.05 \mathrm{mg} / \mathrm{kg}$ every hour. Muscle relaxation was tested regularly and extra doses were administered if necessary. The animals were intubated and respired with $30 \%$ oxygen and $70 \% \mathrm{NO}_{2}$. At the beginning of the sessions, the eyes were dilated with topical administration of one drop of tropicamide and of atropine. Rectal temperature, ECG, $\mathrm{PCO}_{2}$ in the expired air, and arterial oxygen levels were continuously monitored. The animals were placed between two thermal blankets and rectal temperature was maintained at $37.2^{\circ} \mathrm{C}$. Head movements (e.g., caused by respiration) were suppressed by a bite bar and bands.
ERG recordings

ERG recordings were performed using DTL electrodes that were positioned under contact lenses (Titzé, Sion, Switzerland). At the beginning of each session, ocular refraction was measured with the contact lenses in place using the procedure of the confocal scanning laser tomography. The eyes were normally slightly myopic (between +2 and +3 diopters), so that a correction to obtain a sharp retinal image of the stimulus monitor, located at $33 \mathrm{~cm}$ distance, was not necessary in any of the experiments. This was confirmed by the fact that small displacements of the stimulus resulted in detectable changes in the ERG responses (see below). The contact lenses were also used to protect the eyes against desiccation. During a recording session, mfERGs were first recorded from the right eyes and then from the left eyes. A black occluder was used to prevent stimulation of the fellow eyes. The DTL electrode on the stimulated eye was used as active electrode, the DTL electrode on the fellow eye was used as reference electrodes. Ground electrode was a gold-cup electrode positioned on the right temples which were shaved and cleaned. A wire placed in the mouth was connected to an external ground. This additional grounding substantially suppressed intrusion of $50 \mathrm{~Hz}$ noise of the mains.

The ERG signals were amplified $100,000 \times$ and band-pass filtered between 1 and $300 \mathrm{~Hz}$ (Grass amplifiers). In only a few measurements (about $2 \%$ of all measurements) the $50 \mathrm{~Hz}$ intrusion of the mains was large, necessitating the use of a notch filter.

\section{Stimuli}

The ERG responses to $\mathrm{m}$-sequenced flashes without intervals in 103 equal sized $\left(4.7^{\circ}\right)$ hexagons were measured using the VERIS Science 4.0 software. The size of the entire stimulus was approximately $46^{\circ}$. Before a complete measurement was performed, the responses to short $(3 \mathrm{~min})$ sequences were used to reposition the monitor so that the central hexagon was projected onto the macular region. If necessary, this procedure was repeated until the macular response was centered. The main criterion of a well-centered stimulus was evenly distributed response amplitude to the stimuli in the first and second rings. This task could be easily performed 
between one and four repetitions that were normally sufficient to center the monitor satisfactorily. Misalignments of about $1.2^{\circ}$ (less than one centimeter on the monitor) could be detected with the abovedescribed procedure. When the stimulus was centered on the central retina, the responses to $7 \mathrm{~min}$ sequences (m-sequence exponent 15$)$ were measured. The responses were measured to stimuli at three different contrasts: 99\% (luminance of the dark hexagons: $0.5 \mathrm{~cd} / \mathrm{m}^{2}$; luminance of the bright hexagons: $102 \mathrm{~cd} / \mathrm{m}^{2}$ ), $58 \%$ and $31 \%$. The mean luminance was about $51 \mathrm{~cd} / \mathrm{m}^{2}$. The luminance of the dark and bright hexagons were calibrated every recording session with a Minolta LS-110 luminance meter and adjusted if necessary. The measurements were performed with a dim ambient room light. A black funnel-shaped extension between the monitor and the animal blocked the ambient room light. The calibrations were performed before and under identical stimulus conditions as during the measurements.

\section{Data analysis}

In the present article only the first order kernels of the responses are considered. The differences between responses were quantified without any a priori assumptions about the ERG components that are mainly involved. The responses were compared with those measured at equivalent retinal areas in the same eye at the first baseline measurement and in the fellow eyes at the same session.

The differences in response waveform with those measured at the first baseline measurement were quantified by summing the absolute differences at each discrete signal sample during the first $90 \mathrm{~ms}$ of the response. These responses were normalized to the amplitude of P1 (the first positive peak between 20 and $40 \mathrm{~ms}$ after stimulus onset) in the first baseline measurement. Owing to the normalization procedure, the results of the different individual animals and the different retinal locations can be compared. Thus a temporal response change $\left(D_{\text {time }}\right)$ was calculated as follows:

$D_{\text {time }}=\frac{\sum_{i=1}^{x}\left|R_{b, i}-R_{m, i}\right|}{\max \left(R_{b}\right)}$

in which $R_{b}$ is the baseline response and $R_{m}$ is the response at the current recording session; $x$ is the number of the discrete samples of the signal (109 with the used settings). The analysis is based upon changes in the complete waveform. Although this analysis does not consider that certain components may be primarily affected it takes into account the complete ERG trace and can be expected to detect any significant change.

The same equation was used to quantify the response differences between the left and the right eyes (inter-ocular response difference, $D_{\text {eye }}$ ). The differences were normalized to P1 in the noncoagulated eye. The inter-individual variability before laser treatment ( $D_{\text {ind }}$ ) was quantified using the same equation, but now the responses in the right eyes of the six animals were compared with the responses in the right eye of a seventh animal. The maximal response in this animal was also used for normalization. All values of $D$ are dimensionless.

This analysis is not meant to replace any other analysis method that may be more informative on the physiological substrates that are primarily affected by OHT. It is rather meant to be a method which reveals whether there are changes at all. This method may suffice in testing of drugs or therapies or in the screening of the development of a disorder in which the aim is to follow the ERG changes per sé. Furthermore, if information on which ERG components are affected by OHT is needed, a pre-analysis with the above-described method might be helpful, because in those cases in which no differences or changes are revealed it will be difficult to detect changes or differences with other methods. In those cases the component analyses may be confined to those cases in which a difference was found.

In order to study whether retinal regions were differently affected, the responses of the macula (Mac) and the averages of responses in four concentric rings around the macula (R1, R2, R3, and R4) and in four quadrants $(\mathrm{Q} 1, \mathrm{Q} 2, \mathrm{Q}$, and $\mathrm{Q} 4)$ were calculated. The descriptions of the regions are summarized in Fig. 1 in which a monitor view of the responses is presented. The responses shown in Fig. 1 were recorded during a baseline session. The responses of R1, R2, R3, and R4 are the averages of responses to stimuli in $6,12,18$, and 24 hexagons respectively. The rings did not overlap. Responses in quadrant Q1, Q2, Q3, and Q4 are averages of the responses in the inferior nasal quadrant, the inferior temporal, the superior nasal and the superior temporal 


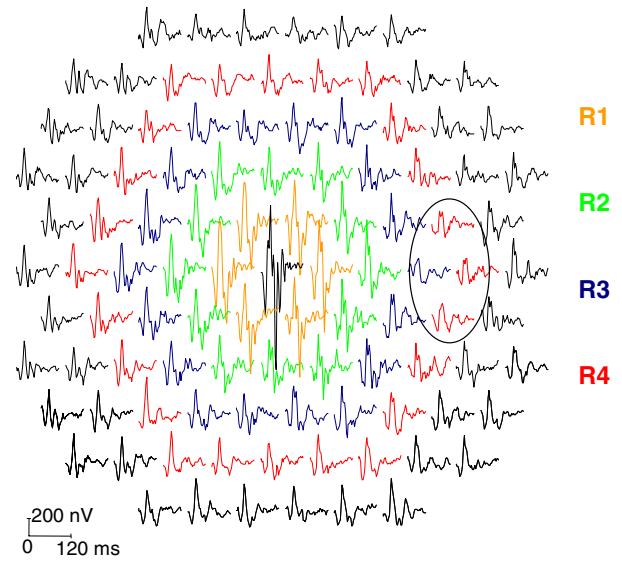

Fig. 1 Definition of the nine used retinal areas. In the left trace array the rings in which the responses are averaged are color coded. The responses are averages of responses in 6 (R1), 12 (R2), 18 (R3) and 24 (R4) hexagons. The quadrants are defined as shown in the right panels. They are averages of responses to

retinal quadrant respectively. The quadrants are averages of the responses to 12 hexagons. The quadrants did not overlap and the responses along the horizontal and vertical meridians were not included in these averages.

\section{Results}

IOPs

In Fig. 2 the IOPs measured at the beginning of each recording session (typically at about 8 a.m.) are displayed as a function of the day of recording. The arrows indicate the days at which the animals underwent laser-coagulation. The IOPs in the treated eyes were increased after one to three laser-coagulations. It was found, however, that the IOPs often decreased at later times during the day. Furthermore, in most animals the IOPs recovered partially 50-100 days after lasercoagulation.

\section{Waveform variability analysis}

In Fig. 1 typical mfERG responses at 99\% stimulus contrast are shown. The macular response (the central response) has a maximal amplitude. The amplitudes are similar for responses of retinal regions with approximately the same distance to the macula
Q2

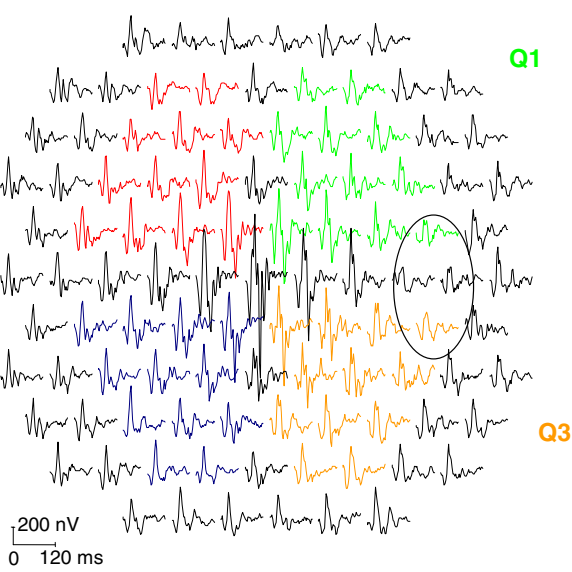

12 hexagons. The central black trace is the macular response (Mac). The four encircled traces display a region with decreased response amplitudes originating from a region close to the optic nerve head

identified by the different rings. Furthermore, the responses at the location of the optic nerve head (the encircled region) have a smaller amplitude. The position of the optic nerve was typically about three hexagons nasally from the macula. Furthermore, the responses display regional variations described before [18, 22, 28, 29]. For instance, high-frequency components can be observed nasally from the macula (this was especially the case at lower contrasts; data not shown).

We first analyzed the response variability by comparing the response waveforms before OHT was induced. For that, we calculated the intra-ocular response differences at the first and second baseline measurements $\left(D_{\text {time }}\right)\{1\}$, the inter-ocular response differences $\left(D_{\text {eye }}\right)$ at the second baseline session $\{2\}$, and the inter-individual response differences $\left(D_{\text {ind }}\right)$ at the second baseline session $\{3\}$ using Eq. 1 .

Figure 3a shows the intra-ocular response differences $\left(D_{\text {time }}\right)$ between the first and the second baseline measurements. The means and standard deviations of $D_{\text {time }}$ across the six different animals are shown separately for the nine defined retinal regions and for the three stimulus contrasts. Figure $3 \mathrm{~b}$ shows two macular responses to a $99 \%$ contrast stimulus measured in the right eyes of one animal (G9) at the two baseline measurements. $D_{\text {time }}$ between the two displayed responses was 20.3, which is about the average for the macular response of all animals (compare with Fig. 3a). The intra-ocular 

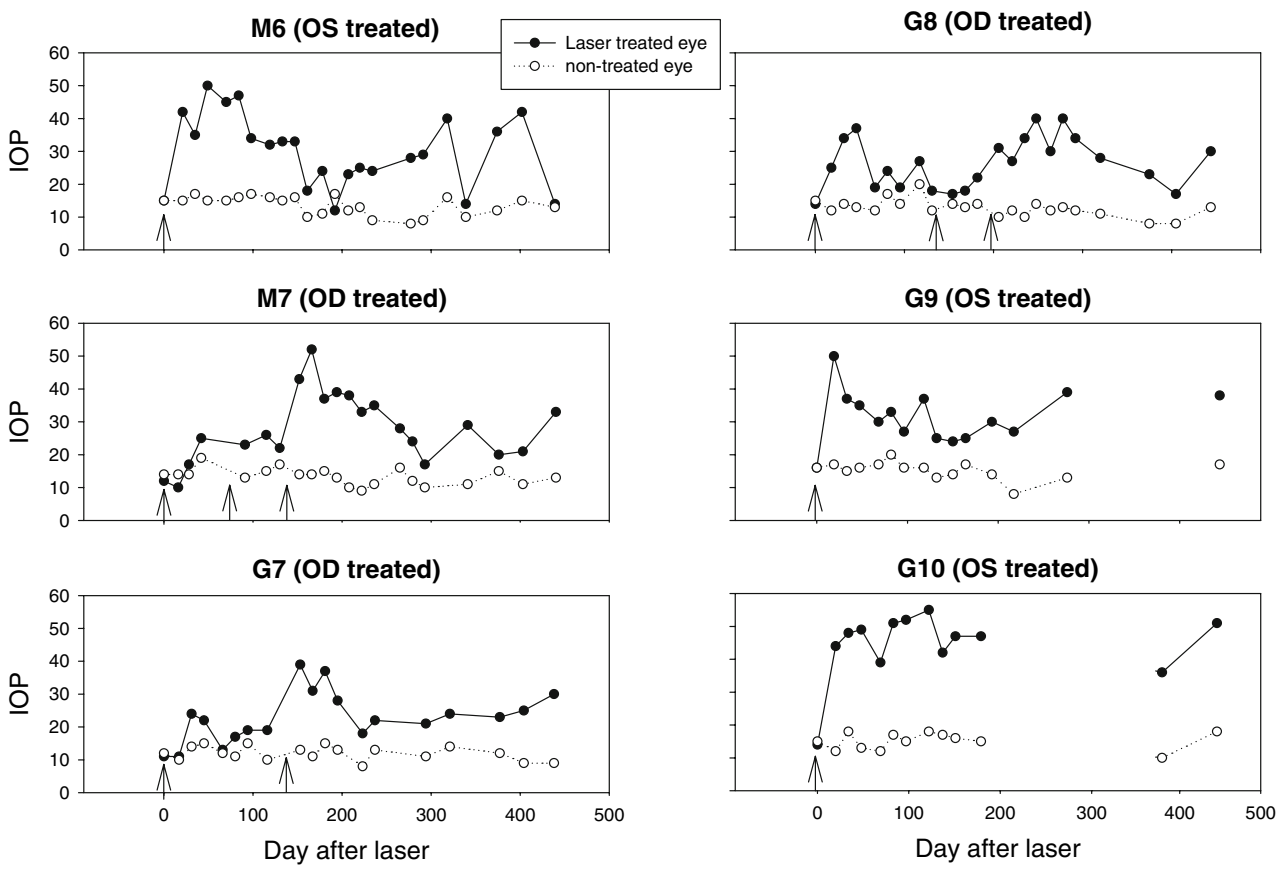

Fig. 2 Intraocular pressures measured in the six monkeys over the period of measurements. The treated eyes display increased IOPs, but sometimes the laser treatment had to be applied more than once, to obtain a clear ocular hypertension. A recovery

over time was generally observed. When the intervals between two measurements were more than 100 days (monkeys G9 and G10) the data points were not connected

differences are largest for the macular response, probably because here the response to the stimulus in only one hexagon is involved which therefore is more sensitive to small misalignments. Moreover, the central hexagon stimulates the retinal region in which the photoreceptor and retinal ganglion cell densities change most dramatically with eccentricity thereby increasing the sensitivity to misalignments. Further, $D_{\text {time }}$ is generally slightly larger for the lower stimulus contrasts probably because of a smaller signal to noise ratio.

In Fig. $3 c$ the means and standard deviations of the inter-ocular response differences $\left(D_{\text {eye }}\right)$ measured at the second baseline session are shown. The differences are larger for the macular response (as in Fig. 3a) and lower stimulus contrasts. $D_{\text {eye }}$ is generally smaller than $D_{\text {time }}$ (compare with Fig. 3a and c), indicating that the response differences between the two eyes within a session is smaller than the response variability in the same eye at different recording sessions (paired $t$-test; $P<0.00001$ ). An example of two macular responses to $99 \%$ contrast stimuli in the two eyes of monkey G9 at the second baseline measurement is shown in Fig. 3d ( $D_{\text {eye }}$ in this example was 7.65).

In Fig. 3e the inter-individual response differences $\left(D_{\text {ind }}\right)$ are displayed separately for the different retinal areas. The responses measured in the right eyes during the second baseline session were compared with those measured in the right eye of monkey G8. A seventh animal was included in this comparison so that the same number of comparisons (six) is used. It can be seen that the inter-individual differences are generally larger than the differences between the responses measured in the same individual (compare with Fig. 3a and c). Assuming that the ERG reflects the functional organization of the retina, this suggests that the functional organization of the retina is similar in the left and right eyes of the same animals but can be more variable in different individuals. However, the quantification of the inter-individual differences is confounded by differences between recording sessions. Nevertheless, the inter-individual differences are significantly larger than the inter-session differences (paired $t$-test $P<0.00001$ ). In Fig. 3f, the macular ERG responses to $99 \%$ contrast stimuli of 
$100 \%$ contrast $31 \%$ contrast

A Comparison between first and second baseline; right eyes
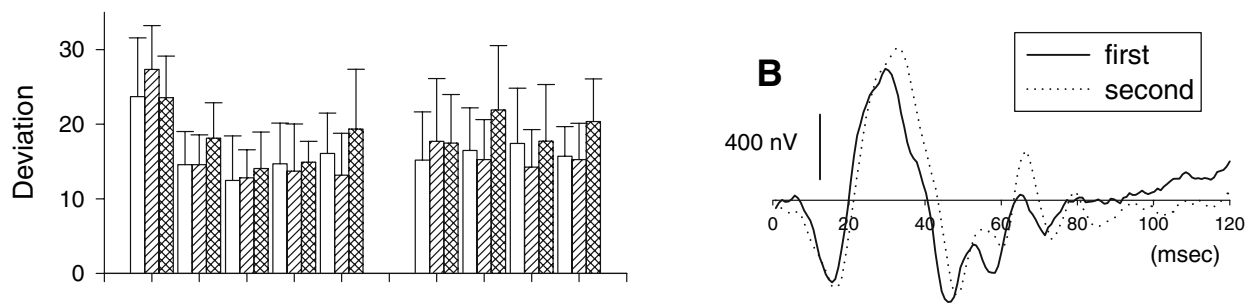

C Comparison between left and right eye; second baseline

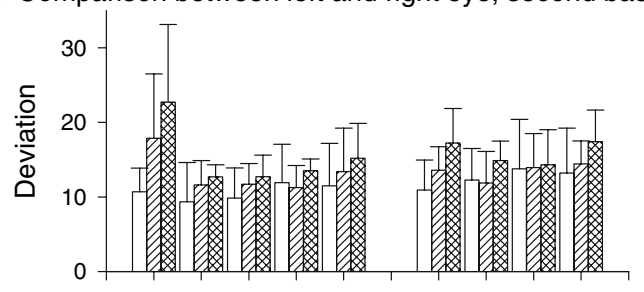

E Comparison the right eyes of six animals with monkey G8
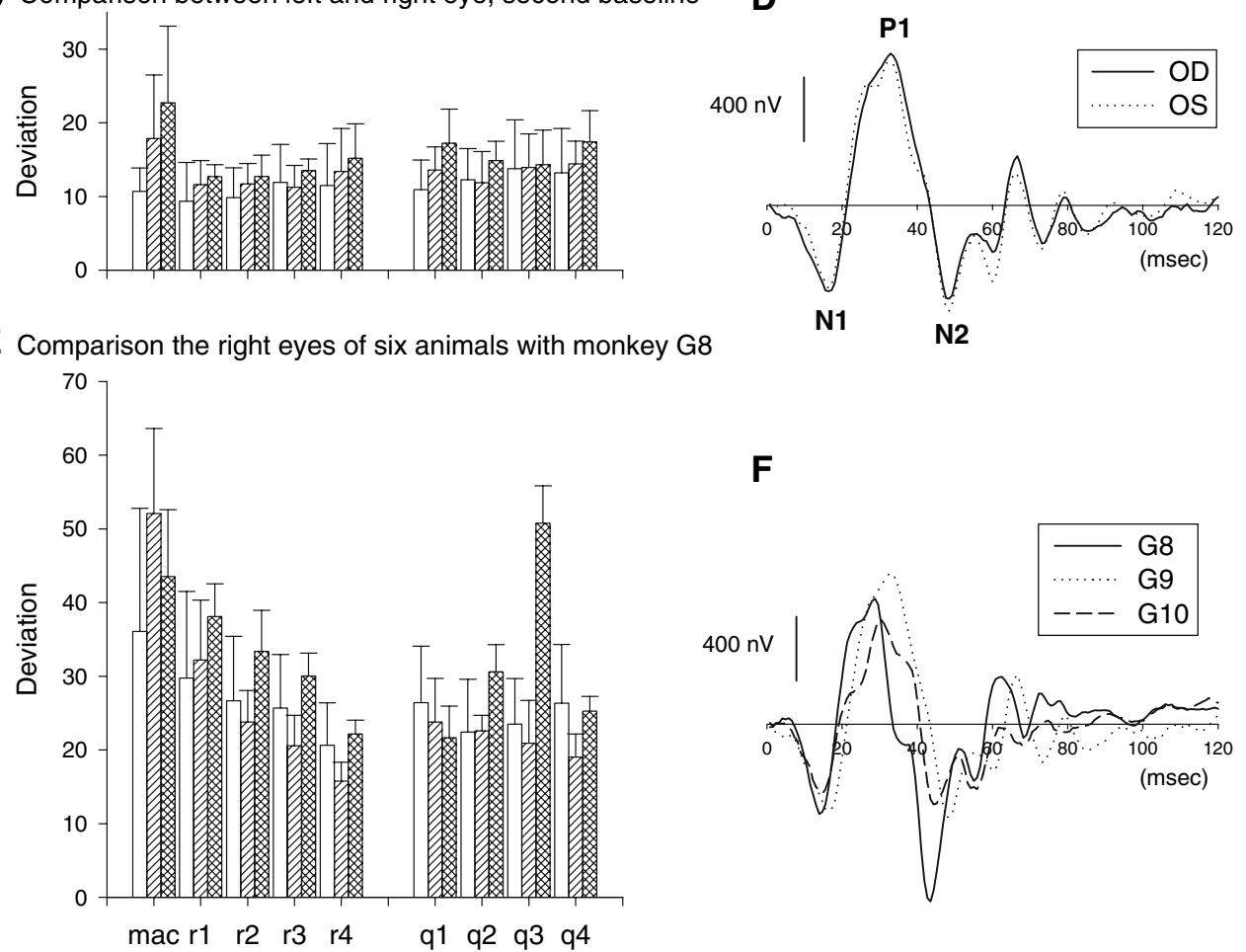

Fig. 3 Differences between signals. (a) The intra-ocular differences between the responses measured at the first and second baseline measurement $\left(D_{\text {time }}\right)$. (b) An example of the macular responses measured at first and second baselines session in monkey G9. (c) The inter-ocular response differences $\left(D_{\text {eye }}\right)$ measured at the second baseline session. (d) Examples of

three animals (G8, G9 and G10) at the second baseline measurement are displayed. The values of $D_{\text {ind }}$ were: 48.3 (G8 vs. G9) and 31.6 (G8 vs. G10).

We now consider the changes in mfERG after experimentally induced ocular hypertension. Examples of the changes of the responses in the macular hexagon and the four rings are displayed in Fig. 4 for three animals. These responses in the OHT and the normotensive eyes were measured at about 180 days measured macular responses in the left and right eyes of monkey G9. The ERG components N1, P1 and N2 are identified in this plot. (e) Inter-individual response differences $\left(D_{\text {ind }}\right)$ measured in the right eyes at the second baseline measurement. (f) Macular responses measured in the three different animals (G8, G9 and G10) during the second baseline session

after the last coagulation so that recovery in IOP has completed. Animal G10 displayed distinct differences between the responses measured in the two eyes. The difference in animal G8 are much smaller and monkey M6 there were no or only few differences.

The most obvious changes were visible in monkey G10. This monkey underwent laser coagulation once. The values of $D_{\text {time }}$ and $D_{\text {eye }}$ are displayed in Fig. 5 as a function of the day of recording. $D_{\text {time }}$ in the 
G10 day 179

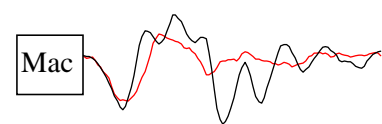

I

R1

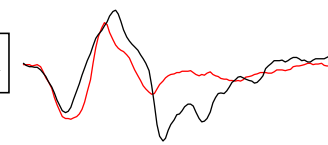
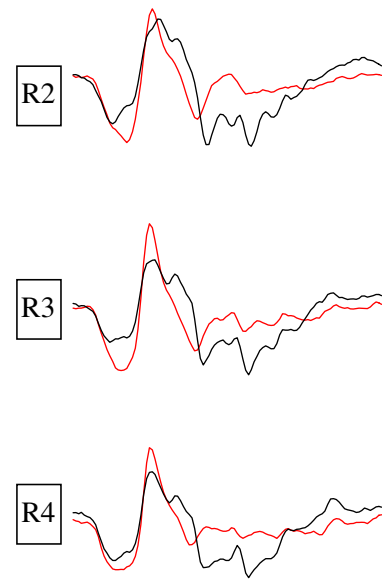

G8 day 375
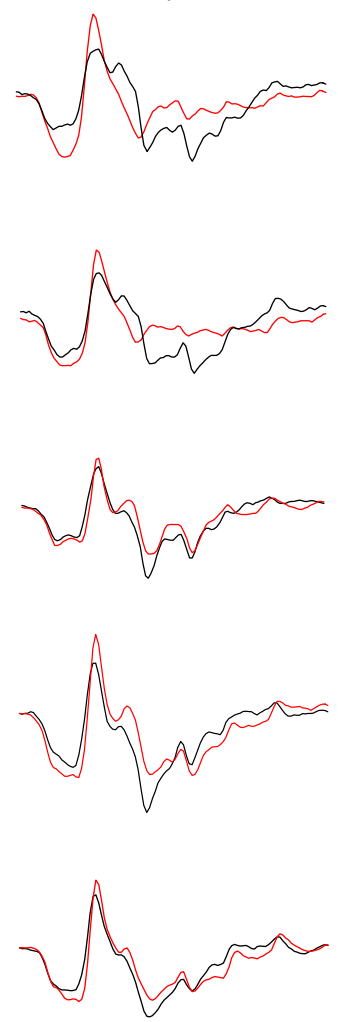

M6 day 178
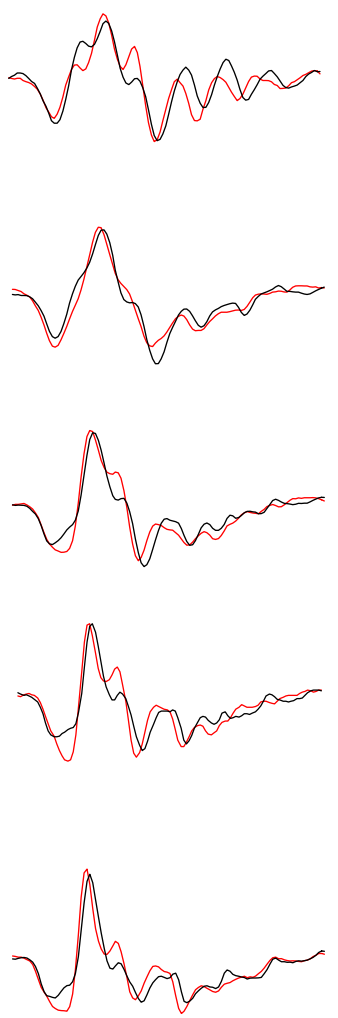

Fig. 4 Original responses to $99 \%$ contrast stimuli measured about 180 days after the last coagulation. The days at which the measurements were performed are given above the traces. In the five rows the ERGs in the macula and rings $1-4$ are displayed. Red traces are the ERGs in OHT eyes. The black traces are the ERGs in non-treated eyes. OHT eyes in G10 and M6: OS; in G8: OD. Vertical bars: $200 \mathrm{nV}$; Horizontal bar: $20 \mathrm{~ms}$. Shown are the responses in a $90 \mathrm{~ms}$ window which is the window used for data analysis

contrasts. In a second series of tests, the effects of laser coagulation, the measured eye and the retinal field on $D_{\text {time }}$ were statistically compared, separately for the different animals and stimulus contrasts. The interaction between laser-coagulation and eye was studied to test differential effects of laser-coagulation on the two eyes. In addition, the combined interaction of laser-coagulation, eye, and retinal location was studied to test the possible differential effect of lasercoagulation at the various retinal fields. For those animals that underwent laser-coagulation more than once, $D_{\text {time }}$ and $D_{\text {eye }}$ before and after the last coagulation were compared. For monkey G8 the values of $D_{\text {time }}$ and $D_{\text {eye }}$ after the last (third) lasercoagulation were compared with those before the second laser-coagulation, because the IOPs and the values of values of $D_{\text {time }}$ and $D_{\text {eye }}$ displayed a gradual increase between these two laser sessions (see Fig. 2 


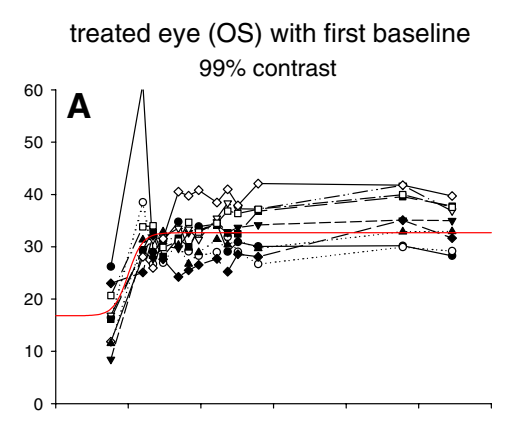

nontreated eye (OD) with first baseline

$99 \%$ contrast
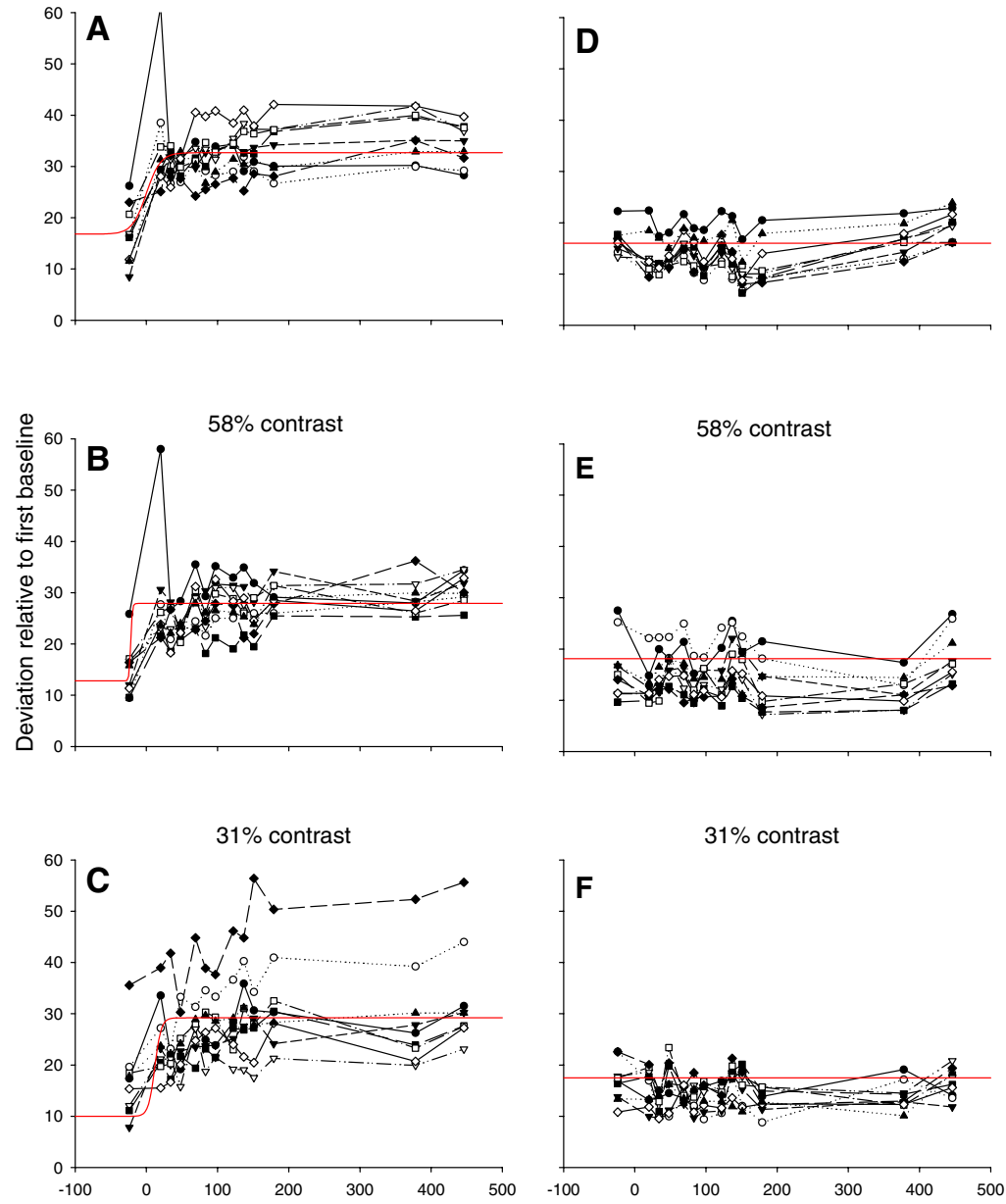
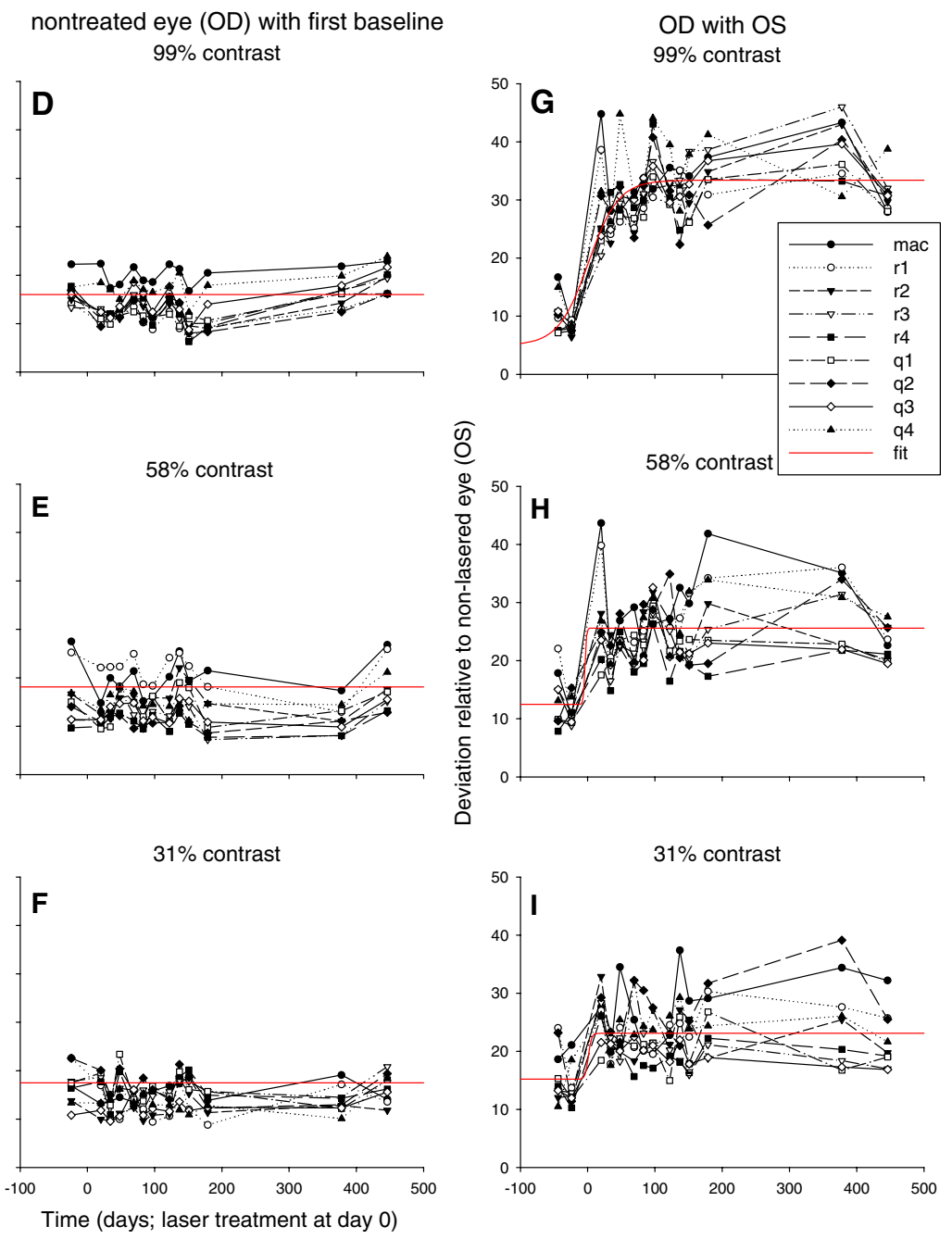

Fig. 5 Changes in the responses measured in monkey G10 caused by laser coagulation. The intra-ocular response differences $\left(D_{\text {time }}\right)$ in the treated eye (OS; left panels) nontreated eye (middle panels) and the inter-ocular response

and below). For all animals and stimulus contrasts, no significant interactions with retinal field were found, showing that the effects of laser-coagulation were similar for the different retinal fields on the value of $D$. That does not mean that using other (such as morphological or psychophysical) techniques and other types of analysis might not reveal any regional differences, but it suggests that the ERGs are changed in a similar manner in the different retinal fields. The results of those tests, in which the differential effects of laser-coagulation on the left and right eyes are compared (thus on $D_{\text {eye }}$ and on the interaction between $D_{\text {time }}$ and eye), are summarized in Table 1 . differences ( $D_{\text {eye }}$; right panels). The results for the responses to $99 \%, 58 \%$ and $31 \%$ contrast stimuli are shown. In each plot the results are shown for the nine defined retinal areas

Laser-coagulation had a significant effect on the ERG responses of three animals (G8, G9, and G10) at all stimulus contrasts. In the remaining animals the effects of laser coagulation were significant for only one stimulus condition, suggesting no or only very mild changes. Furthermore, $D_{\text {eye }}$ is apparently more sensitive to detect laser-induced changes than $D_{\text {time }}$ for which a more indirect test on the interaction between laser-coagulation and eye is necessary.

Since the effects of laser-coagulation were similar for all retinal fields, in the subsequent analysis, the averages of $D_{\text {time }}$ and $D_{\text {eye }}$ of the macula and of rings 1-4 were used. This is a weighted average, because the 
Table 1 Results for the statistical analyses on $D_{\text {time }}$ and on $D_{\text {eye. }}$ Furthermore the estimates of the free parameters of eq. (2) to the data are given

\begin{tabular}{|c|c|c|c|c|c|c|c|c|c|c|c|}
\hline \multirow[t]{2}{*}{ Animal } & \multirow[t]{2}{*}{ Contrast $(\%)$} & \multirow{2}{*}{$\begin{array}{l}\text { Comparison with } 1 \text { st baseline } \\
D_{\text {time }} \text { (interaction between } \\
\text { eye and laser coagulation) }\end{array}$} & \multicolumn{5}{|c|}{ Treated eye } & \multicolumn{4}{|c|}{ Non-treated eye } \\
\hline & & & $D_{0}$ & $a$ & $X_{0}$ & $b$ & & $D_{0}$ & $a$ & $x_{0}$ & $b$ \\
\hline \multirow[t]{3}{*}{ G7 } & 99 & n.s. & 13.1 & 8.6 & 385 & \multicolumn{2}{|c|}{4.3} & 13.4 & 15.2 & 406 & 17.2 \\
\hline & 58 & n.s. & 14.4 & 9.4 & 387 & \multicolumn{2}{|c|}{23.9} & 12.4 & 14.9 & 392 & 8.5 \\
\hline & 31 & n.s. & 16.6 & 13.6 & 377 & \multicolumn{2}{|c|}{35.4} & 12.9 & 16.4 & 395 & 12.8 \\
\hline \multirow[t]{3}{*}{ G8 } & 99 & $P<0.001$ & 7.7 & 11.8 & 91 & \multicolumn{2}{|c|}{59.0} & 15.4 & 0 & - & - \\
\hline & 58 & $P<0.001$ & 11.5 & 9.8 & 136 & \multicolumn{2}{|c|}{37.3} & 14.3 & 0 & - & - \\
\hline & 31 & $P<0.001$ & 19.0 & 10.0 & 216 & \multicolumn{2}{|c|}{4.1} & 20.1 & 0 & - & - \\
\hline \multirow[t]{3}{*}{ G9 } & 99 & $P<0.001$ & 14.8 & 6.7 & -24 & \multicolumn{2}{|c|}{2.0} & 13.1 & 0 & - & - \\
\hline & 58 & $P<0.001$ & 9.4 & 10.8 & -25 & \multicolumn{2}{|c|}{0.2} & 20.1 & 3.5 & - & - \\
\hline & 31 & $P<0.001$ & 20.8 & 10.0 & -23 & \multicolumn{2}{|c|}{1.9} & 29.4 & 4.4 & - & - \\
\hline \multirow[t]{3}{*}{ G10 } & 99 & $P<0.001$ & 16.8 & 15.9 & 0 & \multicolumn{2}{|c|}{10.0} & 16.5 & 0 & - & - \\
\hline & 58 & $P<0.001$ & 12.8 & 15.1 & -22 & \multicolumn{2}{|c|}{1.1} & 18.1 & 0 & - & - \\
\hline & 31 & $P<0.001$ & 10.0 & 19.2 & 11. & \multicolumn{2}{|c|}{4.5} & 17.5 & 0 & - & - \\
\hline \multirow[t]{3}{*}{ M6 } & 99 & n.s. & 17.2 & 0 & - & \multicolumn{2}{|c|}{-} & 18.7 & 1.5 & - & - \\
\hline & 58 & n.s. & 24.5 & 0 & - & \multicolumn{2}{|c|}{-} & 22.5 & 0 & - & - \\
\hline & 31 & n.s. & 20.1 & 0 & - & - & & 23.7 & 0 & - & - \\
\hline M7 & 99 & $P=0.023^{*}$ & 14.4 & 1.1 & - & - & & 19.2 & 0 & - & - \\
\hline & 58 & n.s. & 15.2 & 1.1 & - & - & & 18.6 & 0 & - & - \\
\hline & 31 & n.s. & 16.4 & 2.0 & - & - & & 19.6 & 0 & - & - \\
\hline Animal & Contrast (\%) & Comparison OD-OS $D_{\text {eye }}$ (influen & e of las & coagu & ion) & $D_{0}$ & $a$ & $x_{0}$ & $b$ & & \\
\hline G7 & 99 & n.s. & & & & 12.2 & 1.4 & - & - & & \\
\hline & 58 & n.s. & & & & 19.8 & 0 & - & - & & \\
\hline & 31 & $P=0.001$ & & & & 13.8 & 0.6 & - & - & & \\
\hline G8 & 99 & $P<0.001$ & & & & 14.2 & 7.6 & 120 & 11.2 & & \\
\hline & 58 & $P<0.001$ & & & & 15.8 & 9.2 & 166 & 23.4 & & \\
\hline & 31 & $P<0.001$ & & & & 19.6 & 8.1 & 214 & 0.4 & & \\
\hline G9 & 99 & $P<0.001$ & & & & 11.4 & 6.0 & 1 & 1.0 & & \\
\hline & 58 & $P<0.001$ & & & & 14.0 & 6.7 & 1 & 1.0 & & \\
\hline & 31 & n.s. & & & & 21.8 & 2.4 & - & - & & \\
\hline G10 & 99 & $P<0.001$ & & & & 5.0 & 28.4 & 1 & 22.4 & & \\
\hline & 58 & $P<0.001$ & & & & 12.5 & 13.1 & -4 & 1.1 & & \\
\hline & 31 & $P<0.001$ & & & & 15.2 & 7.9 & 1 & 2.3 & & \\
\hline M6 & 99 & $P<0.001$ & & & & 8.9 & 4.8 & 23 & 1.0 & & \\
\hline & 58 & n.s. & & & & 13.1 & 2.3 & - & - & & \\
\hline & 31 & n.s. & & & & 13.8 & 2.3 & - & - & & \\
\hline M7 & 99 & $P<0.001 *$ & & & & 11.5 & 1.5 & - & - & & \\
\hline & 58 & n.s. & & & & 13.3 & 0 & - & - & & \\
\hline & 31 & n.s. & & & & 13.9 & 0 & - & - & & \\
\hline
\end{tabular}

* Significant influence because $D_{\text {time }}$ in the eye, that was not laser-coagulated (OS), decreased 
responses themselves are means to stimuli in a variable number of hexagons. The response of the macular hexagon is thus weighted 24 times more strongly than a hexagon in ring 4 (which is the average of the responses in 24 hexagons). The reason for choosing this average is that the density of cones is larger in the center. This procedure implies that similar numbers of cones are stimulated by the macular hexagon and by each ring. We estimated the numbers of stimulated cones in the central hexagon and the different rings for human subjects based on anatomical data $[30,31]$. The cone densities in macaques are similar to those in humans [32]. The ratio of stimulated cone numbers was approximately $1: 2: 2.6: 2.3: 3.3$ for the central hexagon and R1-R4. Although these estimates are only rough approximations (also because there can be large inter-individual variability), they show that the numbers of stimulated cones are indeed in the same order of magnitude in the four rings. The central hexagon stimulates only half to one third of the numbers of the cones stimulated in the rings. We nevertheless used this weight because for some ERG components a weighting according to the numbers of stimulated postreceptoral cells (bipolar cell and retinal ganglion cells) might be more appropriate than one reflecting the numbers of stimulated cones. Moreover, because the values of $D$ are not significantly different for different retinal fields, the weightings will only have minor influence on the results.

Using the Excel97 solver routine, the averaged values of $D_{\text {time }}$ and $D_{\text {eye }}$ were fitted with a sigmoidal curve described by the following equation:

$D=D_{0}+\frac{a}{1+e^{-\left(\frac{x-x_{0}}{b}\right)}}$

in which $D$ is the response difference as a function of $x$ which is the date at measurement; $D_{0}, x_{0}, a$ and $b$ are free parameters. Parameter $D_{0}$ is the initial response difference. Parameter $a$ indicates the maximal response difference relative to $D_{0}$ (i.e., the difference of the value of $D$ at the plateau of the sigmoid curve and $D_{0}$ ) and was constrained to positive values, forcing the values of $D$ to increase or remain stable as a function time. $x_{0}$ is the date at which a half maximal response difference was reached. $b$ determines the speed of response change. By using this equation we implicitly assumed that a laser-induced change in the ERGs would cause an increase in response difference over time which will then level off when end stage is reached. Possible subsequent recovery was not considered. Although the function is empirical it gives adequate descriptions and the parameters can easily be interpreted. We constrained the fits by requiring that $D_{0}$ is smaller than 5. The values of $x_{0}$ and $b$ are meaningless when $a$ (the change in ERG response induced by ocular hypertension) is small (approximately <4.5). Furthermore, the accuracy of the estimation of $x_{0}$ is limited to a few days. The accuracy further decreases with decreasing values of $b$ (in the extreme case, when $b$ is very small there is a increase from $D_{0}$ to $D_{0}+a$ between two measurements, so that $x_{0}$ can only be estimated with an accuracy of less than 14 days, depending on the time that elapsed between two measurements). The estimated parameters obtained from the fits are summarized in Table 1 (The values of $x_{0}$ and $b$ were omitted when $a<4.5$ ).

The curves in Fig. 5 are fits of this function to the above-described averages of $D_{\text {time }}$ and $D_{\text {eye }}$ for animal G10. Obviously, $D_{\text {time }}$ in the eye with increased IOP (left panels) and $D_{\text {eye }}$ (right panels) increase after laser coagulation with values between 7.9 and 28.4 ( $a$ in Table 1), whereas $D_{\text {time }}$ in the fellow eye (middle column panels) has not changed. For all the other animals, only the data at $99 \%$ stimulus contrast are shown. In Fig. 6, the results for the two other monkeys (G8, right panels and G9 left panels), that displayed significant changes, are presented. In agreement with the statistical analysis, and similar to the data on monkey G10, $D_{\text {time }}$ in the eyes with OHT (upper panels) and $D_{\text {eye }}$ (lower panels) increased whereas $D_{\text {time }}$ in the fellow eyes (middle row panels) were not altered. However, the changes were smaller than those for G10 (with values of $a$ between 7.6 and 11.8 for G8 and between 2.4 and 10.8 for G9). The statistical analysis showed that the smallest change of $D_{\text {eye }}$ in monkey G9 (by 2.4 at $31 \%$ stimulus contrast) was not significant.

The values of $x_{0}$ correlated well with the time of coagulation and of IOP increase (days 0 for monkeys G9 and G10; between second and third coagulation at days 136 and 193, indicated by the arrows in Fig. 6, for monkey G8). In monkey G8 there are indications that some changes might have occurred after the first laser treatment that resulted in a transient increase in IOP.

The results of the animals which showed no or only mild changes correlated with increased IOP, are shown in Fig. 7 (M7 left, G7 middle column and 


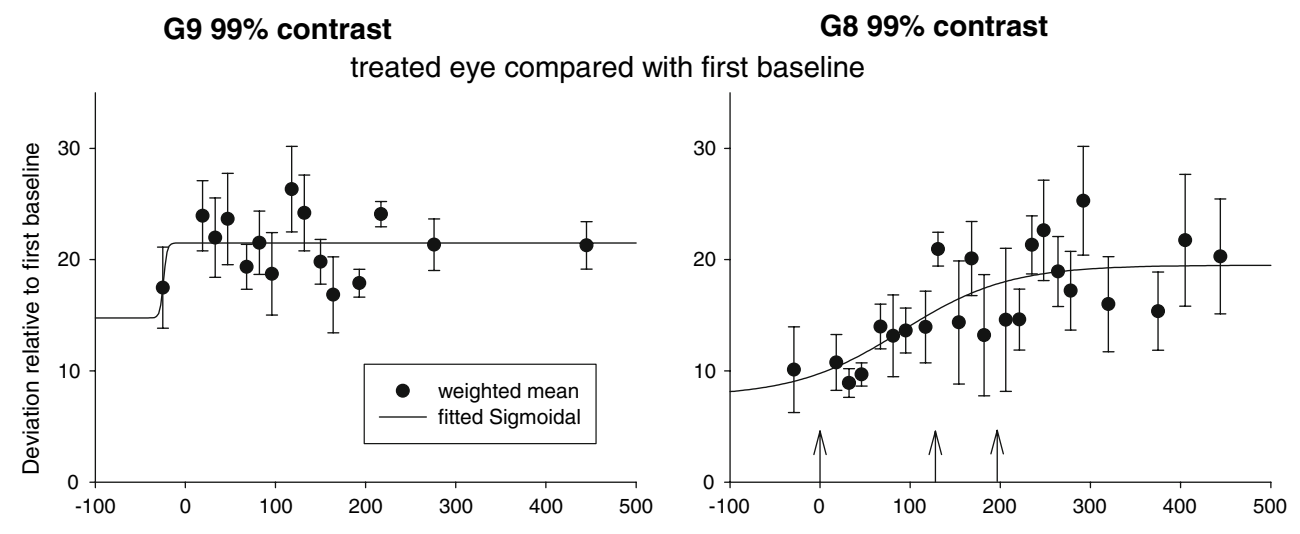

G8 $99 \%$ contrast

non-treated eye compared with first baseline
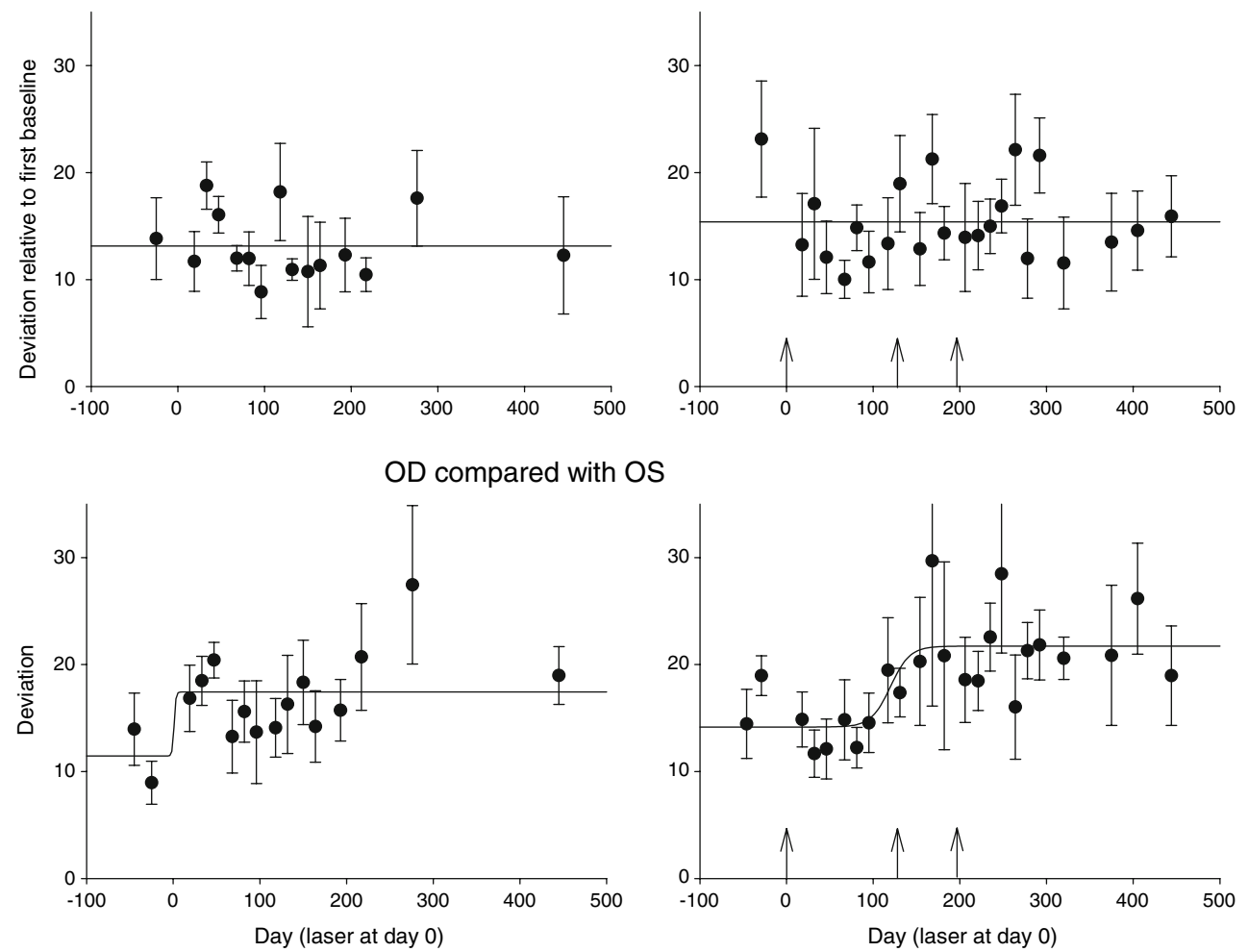

Fig. 6 Averaged waveform changes in the treated and nontreated eyes and the inter-ocular comparison for two monkeys (G9 left panels; G8 right panels) obtained with 99\% contrast stimuli. The data points and error bars are the means and standard deviations of the responses in the macula and

M6 right panels). The arrows indicate the dates of laser coagulation. In none of the cases there are clearly detectable changes. The statistical analysis showed that the increase in $D_{\text {eye }}$ by 4.8 (compare with Table 1) in monkey M6 (lower right panel) is significant. The other changes in $D_{\text {eye }}$ that were statistically significant did not display large changes

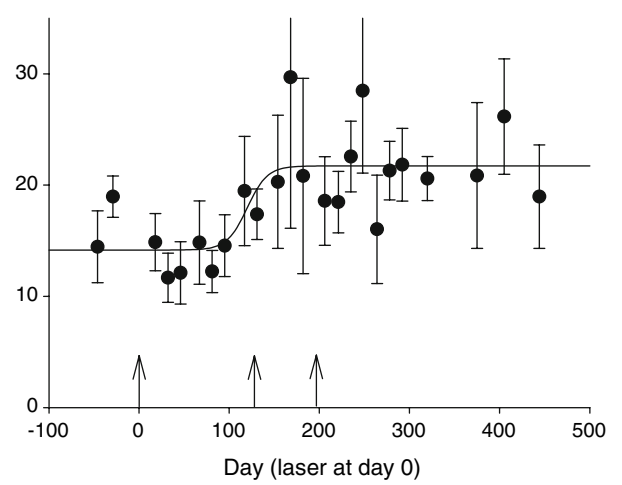

rings 1-4. Although the data are seemingly noisy, a change related with ocular hypertension can be found in the treated eye. The arrows in the right plots indicate the days of laser coagulation. Animal G9 was laser coagulated at day 0. The curves are fits of sigmoid functions to the data

(value for $a$ in $\mathrm{G} 7$ at $31 \%$ contrast: 0.6 ; value for $a$ in M7 at 99\% contrast: 1.5). Taking into account all data, the fits are in agreement with the statistical analysis.

An additional factor that made the analysis less straightforward for monkeys $\mathrm{M} 7$ and $\mathrm{G} 7$ was the fact that during some session the values of $D_{\text {time }}$ were strongly increased in both eyes (indicated by arrow heads). 
G7

treated eye compared with first baseline
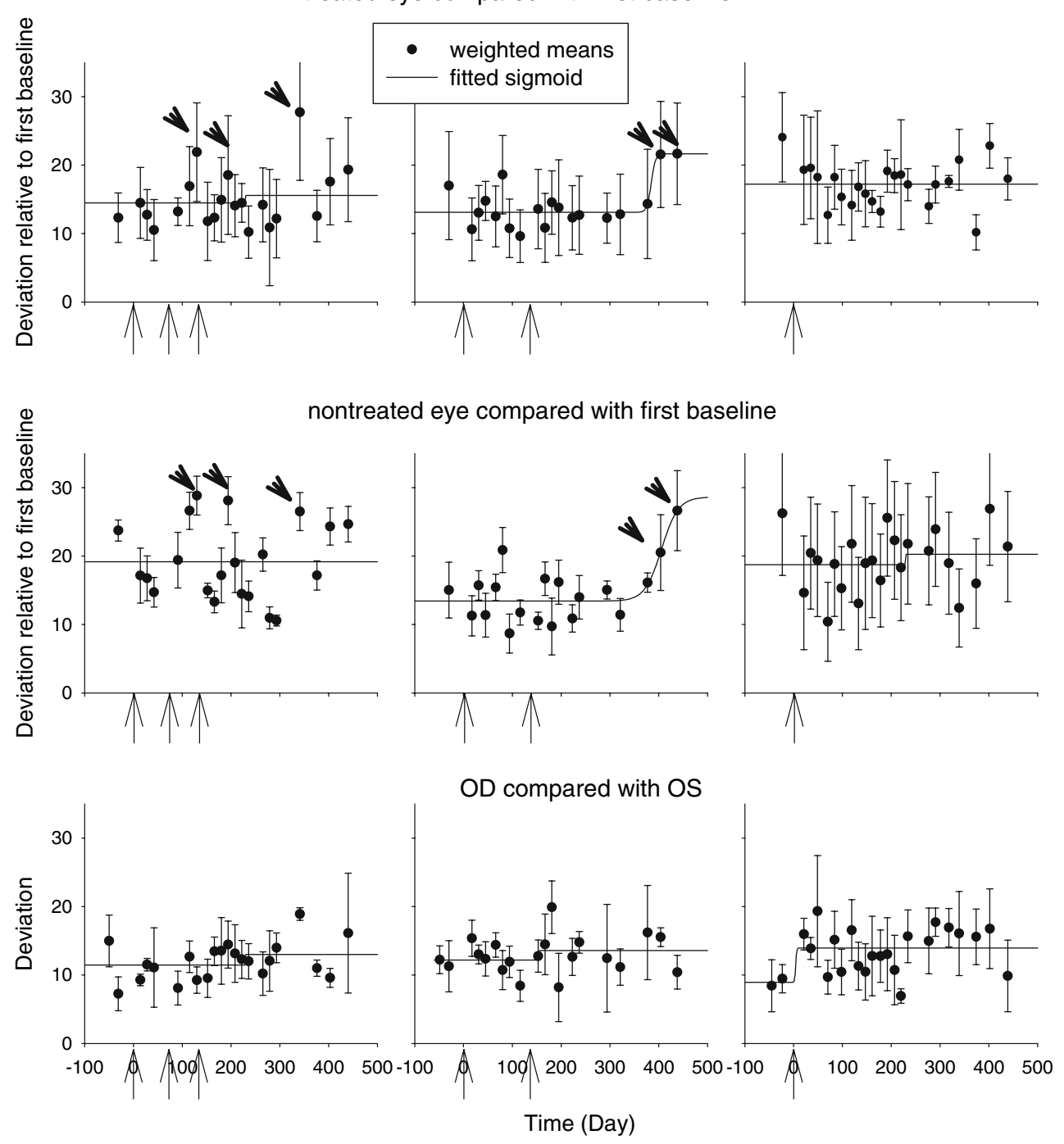

Fig. 7 Response differences in monkeys M7 (left panels), G7 (middle column panels) and M6 (right panels) obtained with the $99 \%$ contrast stimuli. No or only weak response waveform changes, that are related to treatment, can be found. However, large changes were found in both eyes of monkeys M7 and G7 (indicated by arrow heads). These changes were similar in the two eyes, not correlated with ocular hypertension and reversible at least in monkey $\mathrm{M} 7$
However, the values of $D_{\text {eye }}$ were not altered at these days, indicating that similar response changes were present in the two eyes during these sessions. Furthermore, the days of the response changes were not correlated with laser coagulation or a change in IOP. Finally the changes were reversible at least in monkey M7. We, therefore, conclude that the response changes in these animals were not related to laser coagulation and increased IOP.

\section{Discussion}

Variability in the normal retina

The values of $D_{\text {time }}$ are generally small, suggesting that the measurements of ERG responses can be reproducible and indicating that the recording technique and the procedure of centering the stimulus screen can be performed accurately (see also the 
recordings in Figs. 3 and 4). Surprisingly, the interocular ERG responses differences $\left(D_{\text {eye }}\right)$ were even smaller than the intra-ocular response differences at different sessions ( $\left.D_{\text {time }}\right)$. This indicates that the variability, caused by differences in the conditions of the animals and the positions of the electrodes in different sessions, is larger than the differences between the two eyes. $D_{\text {time }}$ was generally small. However, in monkeys M7 and G7 $D_{\text {time }}$ was occasionally strongly increased. This increase was reversible. This suggests that in some animals different ERG response may occur even under seemingly normal circumstances. Possibly, general factors, such as depth of anesthesia, may have influenced the ERG response in these animals. Although these reversible increases in $D_{\text {time }}$ do not occur often, they may pose an important problem in interpreting the data and identifying OHT-induced retinal changes. To be able to take these larger intersession changes into account, it might be necessary to increase the number of baseline measurements and to use an average of the baseline measurements as a reference for calculating $D_{\text {time }}$.

\section{Changes related to ocular hypertension}

All animals displayed prolonged ocular hypertension after one to three laser treatments even though most animals displayed a partial recovery of the IOP during the first 80-100 days after coagulation (Fig. 2). A similar IOP recovery was described before [12]. Not all animals showed laser induced changes in the mfERGs, suggesting that ocular hypertension, even over an extended time, does not always lead to functional changes that can be revealed by mfERG. Such variable results were also obtained before [12]. Nevertheless, animal G10 displays the highest IOPs in the laser treated eye and the largest mfERG changes. In Fig. 8 the values of $a$ (the maximal changes in the mfERG) of individual animals and for the different stimulus contrast conditions, obtained from the fits of Eq. 2, are plotted against the averaged IOP in the laser treated eye (left plots) and against the averaged IOP difference between the OHT and the normotensive eyes (right plots) in the same animal. The IOP measurements more than 100 days after the last laser coagulation were used to calculate the averages. All plots indicate a positive correlation between ERG change and IOP. But for the ERG comparison with the first baseline measurement in the same eye $\left(D_{\text {time }}\right.$; lower panels) the positive correlation is only caused by the large changes in monkey G10. The other animals do not show a correlation. When the comparison between left and right eyes ( $D_{\text {eye }}$; upper panels) were used to quantify the ERG changes, the correlation is clearer especially when the IOP difference between the two eyes is used (upper right plot). We conclude that a comparison between the two eyes may give more reliable results than a comparison in the same eye over time. This conclusion is in agreement with previous observations [12, 20, 21]. The data also show that, although moderate OHT not necessarily leads to functional changes, there is a correlation between IOP and mfERG changes.

From the three monkeys that displayed significant functional changes associated with laser coagulation, only one (G10) showed clear alterations. The changes in the ERG signals in the two other animals were relatively subtle and the values of $D_{\text {time }}$ and $D_{\text {eye }}$ were not larger than the normal inter-individual response differences ( $D_{\text {ind }}$; compare with Fig. $3 \mathrm{e}$ ). Thus a decision that a response is pathologic solely on the basis of the responses without any comparison, either with a condition before induction of ocular hypertension or with a normal fellow eye, is difficult. This is the problem a clinician is facing, because these comparisons are seldom possible. However, an averaged normal ERG waveform could be obtained and a comparison with this waveform may then be performed. Owing to the larger inter-individual variability, the sensitivity of the method to detect OHT induced changed is impaired in comparison with the monkey data described in the present article. However, this problem is not specific for the method described here.

In order to reach the objective of the present study a sensitive method was developed with which changes in the ERGs can be detected. The complete mfERG waveform was considered so that all possible changes could be captured. This method may be useful to detect changes per sé and also to screen the progression of disorder and the success (or absence thereof) of a therapeutic treatment. However, a conclusion about which ERG components were affected and thus on the physiological substrate, that is affected, is not possible. The method may be useful 

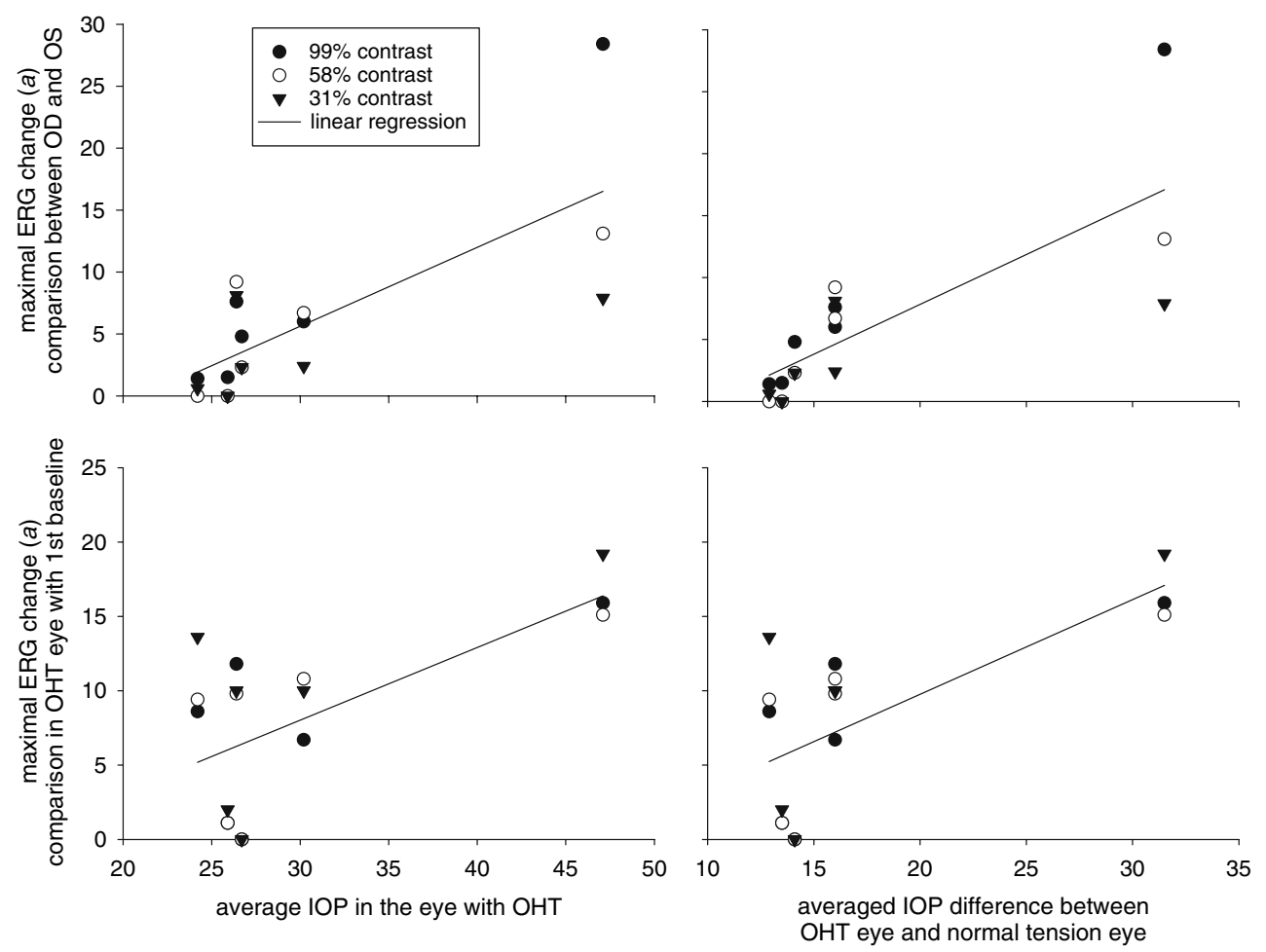

Fig. 8 The waveform change in the mfERG at steady state in Eq. 2 (parameter $a$ ) as a function of the averaged IOP in the OHT eye (left panels) and the difference in averaged IOP between the OHT and normotensive eyes (right panels). The data points represent data from individual animals and from different stimulus contrasts. In the upper panels, the values of

in identifying those ERG data on which other analysis methods can be applied. The disadvantage of the analysis of amplitudes and delays of ERG components can be that only a limited number of data of the ERG recordings are used which under certain circumstances may result in an increased variability of the data and, therefore, a decreased sensitivity to detect changes. This may happen when two components, such as P1 and oscillatory potentials, appear superimposed in the signal. Small changes in the relative locations of the two components may lead to large changes in the estimated amplitude and delay of P1. A compromise can be that analyses of waveform differences, as described in the present article, are performed within predefined time windows. The first $20 \mathrm{~ms}$ of the ERG may give information about the change in the responses directly driven by the photoreceptors and early postreceptoral mechanisms [33, 34]. The next 20-30 ms might give information of changes in the P1 component, and the analysis parameter $a$ were obtained from the comparison between left and right eyes $\left(D_{\text {eye }}\right)$. For the lower panels, the ERG data in the eye with OHT were compared with the 1 st baseline measurement $\left(D_{\text {time }}\right)$. The positive correlation is clearer when $D_{\text {eye }}$ and when the IOP differences rather than the IOP in the OHT eyes were used

within the time window starting about 40-50 ms after stimulation could detect change in the $\mathrm{N} 2$ component. Of course, once components are identified that are sensitive to OHT (e.g., the optic nerve head component, OPs, particular frequency components or the photopic negative response $[4,18,19,28,29])$ concentrating data analysis upon these components may be more sensitive.

We observed that in monkey G10 oscillatory responses diminished which is in agreement with previous observations [9]. Furthermore in G10 a decrease in the amplitude of a negative component at $40-80 \mathrm{~ms}$ is agreement with observations that the photopic negative response in the Ganzfeld ERG is diminished in animals with experimental glaucoma $[6,19]$ as well as in glaucoma patients [4].

Acknowledgments The authors wish to thank Céline Cojean, Isabelle Questel and Emanuel Faure for technical support, Evelyn Rauscher for organizational support and Leopold 
Schmetterer and Michael Bach for their comments on previous versions of the manuscript.

\section{References}

1. Bach M, Hiss P, Rover J (1988) Check-size specific changes of pattern electroretinogram in patients with early open-angle glaucoma. Doc Ophthalmol 69:315-322

2. Hood DC, Greenstein V, Holopigian K, Bauer R, Firoz B, Liebman JM, Odel JG, Ritch R (2000) An attempt to detect glaucomatous damage to the inner retina with the multifocal ERG. Invest Ophthalmol Vis Sci 41:1570-1579

3. Bach M (2001) Electrophysiological approaches for early detection of glaucoma. Eur J Ophthalmol 11:S41-S49

4. Viswanathan S, Frishman LJ, Robson JG, Walters JW (2001) The photopic negative response of the flash electroretinogram in primary open angle glaucoma. Invest Ophthalmol Vis Sci 42:514-522

5. Holder GE (2001) Pattern electroretinography (PERG) and an integrated approach to visual pathway diagnosis. Prog Retin Eye Res 20:531-561

6. Viswanathan S, Frishman LJ, Robson JG (2000) The uniform field and pattern ERG in macaques with experimental glaucoma:removal of spiking activity. Invest Ophthalmol Vis Sci 41:2797-2810

7. Graham SL, Drance SM, Chauhan BC, Swindale NV, Hnik P, Mikelberg FS, Douglas GR (1996) Comparison of psychophysical and electrophysiological testing in early glaucoma. Invest Ophthalmol Vis Sci 37:2651-2662

8. Sutter EE, Tran D (1992) The field topography of ERG components in man-I. The photopic luminance response. Vision Res 32:433-446

9. Bearse MA, Sutter EE, Sim D, Stamper R (2005) Glaucomatous dysfunction revealed in higher order components of the electroretinogram. Vision Science and its Application, OSA Technical Digest Series, vol 1, pp 104-107

10. Frishman LJ, Saszik S, Harwerth RS, Viswanathan S, Li Y, Smith III EL, Robson JG, Barnes G (2000) Effects of experimental glaucoma in macaques on the multifocal ERG. Doc Ophthalmol 100:231-251

11. Palmowski AM, Allgayer R, Heinemann-Vemaleken B (2000) The multifocal ERG in open angle glaucoma - A comparison of high and low contrast recordings in highand low-tension open angle glaucoma. Doc Ophthalmol 101:35-49

12. Hare WA, Ton H, Ruiz G, Feldmann B, Wijono M, WoldeMussie E (2001) Characterization of retinal injury using ERG measures obtained with both conventional and multifocal methods in chronic ocular hypertensive primates. Invest Ophthalmol Vis Sci 42:127-136

13. Fortune B, Bearse MA, Cioffi GA, Johnson CA (2002) Selective loss of an oscillatory component from temporal retinal multifocal ERG responses in glaucoma. Invest Ophthalmol Vis Sci 43:2638-2647

14. Raz D, Seeliger MW, Geva AB, Percicot CL, Lambrou GN, Ofri R (2002) The effect of contrast and luminance on mfERG responses in a monkey model of glaucoma. Invest Ophthalmol Vis Sci 43:2027-2035
15. Raz D, Perlman I, Percicot CL, Lambrou GN, Ofri R (2003) Functional damage to inner and outer retinal cells in experimental glaucoma. Invest Ophthalmol Vis Sci 44:3675-3684

16. Marx MS, Podos SM, Bodis-Wollner I, Lee P-Y, Wang RF, Severin C (1988) Signs of early damage in glaucomatous monkey eyes: low spatial frequency losses in the pattern ERG and VEP. Exp Eye Res 46:173-184

17. Harwerth RS, Crawford ML, Frishman LJ, Viswanathan S, Smith EL III, Carter C (2002) Visual field defects and neural losses from experimental glaucoma. Prog Retin Eye Res 21:91-125

18. Rangaswamy NV, Zhou W, Harwerth RS, Frishman LJ (2006) Effect of experimental glaucoma in primates on oscillatory potentialy of the slow-sequence mfERG. Invest Ophthalmol Vis Sci 47:753-767

19. Viswanathan S, Frishman LJ, Robson JG, Harwerth RS, Smith EL III (1999) The photopic negative response of the macaque electroretinogram: reduction by experimental glaucoma. Invest Ophthalmol Vis Sci 40:1124-1136

20. Fortune B, Bui BV, Cull G, Wang L, Cioffi GA (2004) Inter-ocular and inter-session reliability of the electroretinogram photopicnegative response (PhNR) in non-human primates. Exp Eye Res 78:83-93

21. Bui BV, Fortune B, Cull G, Wang L, Cioffi GA (2003) Baseline characteristics of the transient pattern electroretinogram in non-human primates: inter-ocular and intersession variability. Exp Eye Res 77:555-566

22. Hood DC, Greenstein V, Frishman LJ, Holopigian K, Viswanathan S, Seiple W, Ahmed J, Robson JG (1999) Identifying inner retinal contributions to the human multifocal ERG. Vision Res 39:2285-2291

23. Polska EA, Doelemeyer A, Unterhuber A, Schmetterer L, Drexler W, Lambrou GN (2004) Early morphological changes in non-human primate eyes with laser-induced ocular hypertension assessed with ultrahigh-resolution optical coherence tomography, scanning laser polarimetry and scanning laser tomography. Invest Ophthalmol Vis Sci Suppl 44:2176

24. Doelemeyer A, Polska EA, Schmid P, Unterhuber A, Sattman H, Hermann B, Lambrou GN, Drexler W (2004) Correlation of in vivo ultrahigh-resolution optical coherence tomography with histological sections in non-human primate eyes. Invest Ophthalmol Vis Sci Suppl 44:2202

25. Polska EA, Doelemeyer A, Kremers J, Unterhuber A, Schmetterer L, Drexler W, Lambrou GN (2005) Morphological and functional assessment of early changes in the macular region and the optic nerve head of ocular hypertensive non-human primates. Invest Ophthalmol Vis Sci Suppl 45:3512

26. Kommomen B, Hyvätti E, Dawson WW (2007) Propofol modulates inner retina function in beagles. Vet Ophthalmol 10:76-80

27. Tanskanen $P$, Kylmä $T$, Kommomen B, Karhunen $U$ (1996) Propofol influences the electroretinogram to a lesser degree than thiopentone. Acta Anaesthesiol Scand 40: 480-485

28. Sutter EE, Bearse MA (1999) The optic nerve head component of the human ERG. Vision Res 39:419-436

29. Zhou W, Rangaswamy NV, Ktonas P, Frishman LJ (2007) Oscillatory potentials of the slow-sequence multifocal 
ERG in primates extracted using the Matching Pursuit method. Vision Res 47:2021-2036

30. Murray IJ, Parry NRA, Kremers J, Stepien MW, Schild A (2004) Photoreceptor topography and cone specific electroretinograms. Vis Neurosci 21:231-235

31. Curcio CA, Sloan KR, Kalina RE, Hendrickson AE (1990) Human photoreceptor topography. J Comp Neurol 292:497-523

32. Goodchild AK, Ghosh KK, Martin PR (1996) Comparison of photoreceptor spatial density and ganglion cell morphology in the retina of human, macaque monkey, cat, and the marmoset Callithrix jacchus. J Comp Neurol 366: $55-75$

33. Sieving PA, Murayama K, Naarendorp F (1994) Push-pull model of the primate photopic electroretinogram: a role for hyperpolarizing neurons in shaping the b-wave. Vis Neurosci 11:519-532

34. Hood DC, Frishman LJ, Saszik S, Viswanathan S (2002) Retinal origins of the primate multifocal ERG: implications for the human response. Invest Ophthalmol Vis Sci 43:1673-1685 